make the study suitable for paediatric implementation included using 'Monkey' - a cartoon character with varying facial expressions. For example, 'Monkey' was linked to a Likert scale, facilitating children to independently respond to questions. Information sheets containing details of the study were handed to parents/carers on the day of their child's procedure. Posters in recovery and business cards to take home displaying the website URL were used to boost response rate. Satisfaction rates were calculated based on patient and parent/carer response to the adapted FFT. Answers to qualitative questions were manually analysed in a thematic approach to identify recurring themes.

Results 22 questionnaires were completed by parents, with 13 patient sections being completed, over 8 months. Parental and patient satisfaction rates were high, at $100 \%$ and $92 \%$ respectively. Attentive, informative and friendly staff were the main justifications for their positive feedback. Suggestions for improvement were limited, with no dominant themes being detected.

Conclusion There were overwhelmingly high satisfaction rates from both the parent and patient perspective, with minimal suggestion for improvements. To strengthen conclusions further data needs collecting. The acceptability by both patients and parents/carers means the resources created in this study have potential to be used across multiple departments and multiple paediatric centers.

\section{G155(P) PROLONGED JAUNDICE CLINIC: ARE WE DOING TOO MANY TESTS?}

${ }^{1} \mathrm{SF}$ Beebeejaun, ${ }^{2} Y$ Young, ${ }^{1} \mathrm{~A}$ Jain. 'Paediatric Department, Royal London Hospital, London, UK; ${ }^{2}$ Medical School, Barts and the London School of Medicine and Dentistry, London, UK

\subsection{6/archdischild-2020-rcpch.126}

Background Prolonged jaundice is common and in the majority of cases is benign and self-resolves. The prolonged jaundice screen aims to rule out serious causes of prolonged jaundice, such as biliary atresia. In our prolonged jaundice clinic (PJC) we test for FBC, blood film, G6PD, TFTs, LFTs, split bilirubin and urine MC\&S. Babies are often being brought back for repeat tests due to insufficient bloods or incidental findings. The process is time consuming, resource consuming and anecdotally resulted in poor patient satisfaction.

Aim We aimed to reduce the number of investigations and potentially unnecessary repeat tests being carried out in the PJC.

Method A retrospective review was conducted of babies seen in the PJC over 13 months. We collected data on investigation results, repeat tests (and reasons for these) and what pathologies were ultimately picked up.

Results 212 patients were seen in the PJC in the study period. $14 \%$ had a bilirubin result greater than $200 \mathrm{mmol} / \mathrm{l}$. 1 baby had a conjugated bilirubin above $25 \mathrm{mmol}$ or greater than $10 \%$ of total bilirubin. Despite this, 68 babies (32\%) had at least one repeat test carried out, with some having multiple repeats. A total of 156 repeat tests were carried out. Out of the 212 babies seen 6 babies (2.8\%) with pathology were detected: 1 autoimmune neutropenia, 3 G6PD deficiency, 1 intra-hepatic jaundice and 1 e-coli UTI requiring treatment with oral antibiotics only.

Conclusion There was a wide variation in how results were interpreted and hence the reasons for repeating/not repeating tests. A large number of babies did not have high bilirubin results but were having repeat tests done for either insufficient bloods or incidental findings in other parts of the screen.

Plan: We have written a new guideline and proposed a 2 tier investigation pathway with all babies having 'tier 1' investigations (FBC and split bilirubin) when initially seen in PJC, but only those with abnormalities in this initial screen going on to have 'tier 2' investigations (repeat tier 1 investigations in addition to reticulocytes, film, group and DAT, LFTs and urine $\mathrm{MC} \& S)$.

\section{G156(P) IMPLEMENTATION OF A MULTIDISCIPLINARY SAFETY BRIEFING IN A TERTIARY PAEDIATRIC CARDIOTHORACIC TRANSPLANT UNIT}

L Dhugga, M Parameswaran, D Blundell, A Khushnood. Paediatric Cardiology, Freeman Hospital, Newcastle upon Tyne, UK

\subsection{6/archdischild-2020-rcpch.127}

Aim The aim of this project was to provide a place for pandepartmental communication that could improve efficiency, safety and patient care in a tertiary paediatric cardiothoracic unit.

Method The unit deals with complex cases involving many disparate teams involved in providing care to each patient, with frequent movement of children between wards and theatres. There was no structured forum for communicating important information across the department, for example staffing shortages, prescribing errors and significant events.

An initial survey was performed assessing the need for better communication across the multi-disciplinary team. $90 \%$ of respondents could think of an incident relating to ineffective communication, and $85 \%$ felt that that incident could have been avoided with better communication. $80 \%$ of respondents felt that a weekly safety briefing would improve communication.

A safety briefing was designed to be delivered in person at a weekly meeting, covering four key areas: Staffing, Awareness, Fix and Events (SAFE). Staff were encouraged to contribute via email or to approach us in person with information to add.

The safety briefing was delivered in person by a junior doctor at a weekly departmental meeting. The verbal delivery was supported by a single projected slide using the SAFE acronym to summarize the briefing, which was then circulated via email to capture those who were unable to attend.

Results A repeat survey was given to the multi-disciplinary team after eight weeks of delivering the briefing. $80 \%$ of respondents thought that the briefing was valuable and should continue in its current format. Recommendations to change included adding clinic availability and including more staffing groups in the email circulation.

Conclusion Using the change model and staff engagement, the aim is for safety briefings to become embedded in our routine. We demonstrate how to implement change through a grassroots process, using a junior led briefing to improve vigilance, safety and communication. The safety briefing was continued by rotating junior doctors but subsequently stopped, highlighting that even when change is introduced with enthusiasm, it can be difficult to maintain. 